TRANSACTIONS OF THE

AMERICAN MATHEMATICAL SOCIETY

Volume 356, Number 4, Pages 1271-1280

S 0002-9947(03)03429-9

Article electronically published on November 4, 2003

\title{
SPLITTING CRITERIA FOR HOMOTOPY FUNCTORS OF SPECTRA
}

\author{
PHICHET CHAOHA
}

This paper is dedicated to the author's beloved wife and daughter: Cherry and Cheese

\begin{abstract}
We explore the interaction between the Taylor tower and cotower, as defined in deriving calculus with cotriples and dual calculus for functors to spectra of functors of spectra. This leads to new splitting criteria which generalize the results in dual calculus for functors to spectra.
\end{abstract}

\section{INTRODUCTION}

Calculus of homotopy functors was first developed by Thomas Goodwillie in the late 1980's (see [2], 3] and 4]). It was an attempt to approximate a homotopy functor (of spaces or spectra) by the so-called Taylor tower of universally $n$-excisive functors (see Figure 1). This theory was recently proved to be very useful in studying many well-known homotopy functors such as the identity functor of spaces (i.e., unstable homotopy theory) and K-theory.

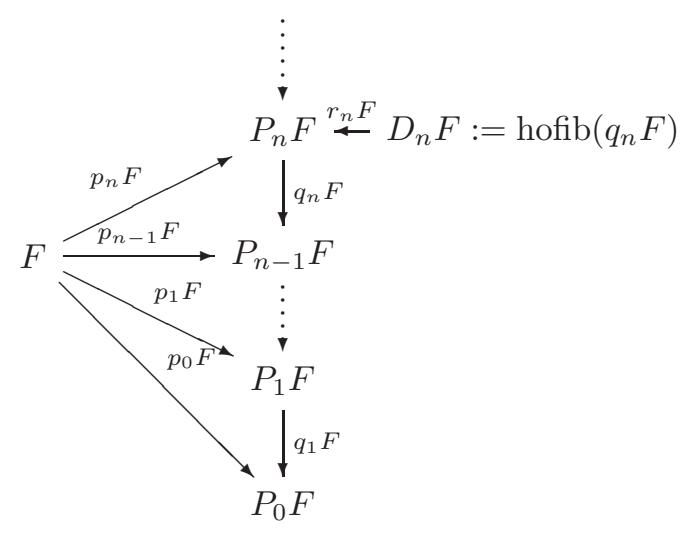

Figure 1. Goodwillie's Taylor tower

Recently, in [5], Brenda Johnson and Randy McCarthy gave another construction (using the theory of cotriples) of the Taylor tower of functors from pointed categories with finite coproducts to abelian categories. However, their tower in-

Received by the editors August 6, 2001.

2000 Mathematics Subject Classification. Primary 55P65.

Key words and phrases. Homotopy functors, calculus of functors.

(C)2003 American Mathematical Society 


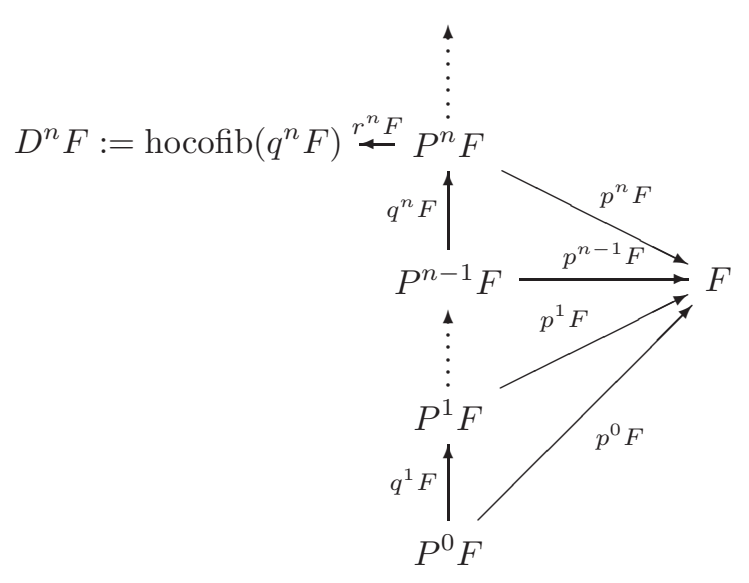

Figure 2. McCarthy's cotower

volves universally degree $n$ functors instead of universally $n$-excisive functors, and the construction can be readily carried on to the case where the target category is the closed model category of spectra (with stably weak equivalences). The picture of their tower looks exactly like the one defined by Goodwillie in [4].

An advantage of Johnson-McCarthy's Taylor tower is that it is dualizable. Therefore, in [6], Randy McCarthy constructs the so-called Taylor cotower (see Figure 2) of functors from pointed categories with finite products to the category of spectra, and he uses it to study the splitting phenomena of homotopy functors of spectra. It turns out that, in the case of homotopy functors of spectra, the theories of Taylor tower and cotower are equivalent if all Tate obstructions (i.e., Tate $\left(\widetilde{\perp}_{n} F ; \Sigma_{n}\right)$ in Definition 1.3 below) vanish. Therefore, it is natural to ask if there is a relationship between the two theories through those obstructions. In this paper, we give a clear answer to the problem by introducing a new construction (originally developed in 1]) which relates $P_{n} F$ (respectively, $P^{n} F$ ) to $P_{n-1} F$ (respectively, $P^{n-1} F$ ) and Tate obstructions. This construction leads to new splitting criteria which generalize the results in [6], and sheds some light on new obstructions for splitting of both tower and cotower.

Throughout this paper, we will assume the reader to be familiar with notations, constructions and properties of calculus and dual calculus developed in [5] and [6]. We will also denote the closed model category of spectra (with stably weak equivalences) by Spec. All natural maps and commutative diagrams of functors to Spec are actually taken in Spec upon an evaluation at a certain spectrum. Therefore, it is immediate that

- (homotopy) fibration and (homotopy) cofibration sequences of functors are equivalent.

- (homotopy) pullback and (homotopy) pushout squares of functors are equivalent.

- (homotopy) cartesian and (homotopy) co-cartesian cubes of functors are equivalent. 
There are many reasons for assuming the source category to be the category of spectra instead of an arbitrary category with finite products and coproducts. Some of them are (see [6] for details), for a homotopy functor $F:$ Spec $\rightarrow$ Spec,

- $c r_{n} F \simeq c r^{n} F$ (equivariantly).

- $F$ is degree $n$ iff $F$ is codegree $n$.

Before leaving this section, we need to define some new terms.

Definition 1.1. The homotopy functor $F: S p e c \rightarrow S p e c$ is called

- $k$-homogeneous if $P_{k} F \simeq *$.

- $k$-cohomogeneous if $P^{k} F \simeq *$.

Remark 1.2. It is easy to show that

- if $F$ is $k$-homogeneous, $P_{i} F \simeq *$ for all $i \leq k$.

- if $F$ is $k$-cohomogeneous, $P^{i} F \simeq *$ for all $i \leq k$.

Therefore, (co)homogeneous degree $n$ functors in the sense of Johnson-McCarthy are exactly $(n-1)-($ co)homogeneous degree $n$ functors in our new definitions.

Definition 1.3. Let $\Delta: S p e c \rightarrow S p e c^{\times n}$ be the diagonal functor, $c r_{n}$ the $n^{\text {th }}$-cross effect, $c r^{n}$ the $n^{\text {th }}$-cocross effect, and $t$ the Tate map (see [6] for details). For any homotopy functor $F: S p e c \rightarrow S p e c$, we define

- $\widetilde{\perp}_{n} F:=\left(D_{1}\right)^{(n)} c r_{n} F \circ \Delta$.

- $\widehat{\perp}_{n} F:=\left(D^{1}\right)^{(n)} c r_{n} F \circ \Delta$.

- $\operatorname{Tate}\left(\widetilde{\perp}_{n} F ; \Sigma_{n}\right):=\operatorname{hocofib}\left(\widetilde{\perp}_{n} F_{h \Sigma_{n}} \stackrel{t}{\rightarrow} \widetilde{\perp}_{n} F^{h \Sigma_{n}}\right)$.

- $\operatorname{Tate}\left(\widehat{\perp}_{n} F ; \Sigma_{n}\right):=\operatorname{hocofib}\left(\widehat{\perp}_{n} F_{h \Sigma_{n}} \stackrel{t}{\rightarrow} \widehat{\perp}_{n} F^{h \Sigma_{n}}\right)$.

In terms of the above definition, it follows that $D_{n} F \simeq \widetilde{\perp}_{n} F_{h \Sigma_{n}}$ and $D^{n} F \simeq$ $\widehat{\perp}_{n} F^{h \Sigma_{n}}$. Moreover, we have the following theorem where the detailed proof can be found in $[5]$ and $[6]$.

Theorem 1.4. For a homotopy functor $F:$ Spec $\rightarrow$ Spec, we have

(1) $\widetilde{\perp}_{n} F_{h \Sigma_{n}}$ and $\widehat{\perp}_{n} F_{h \Sigma_{n}}$ are $(n-1)$-homogeneous degree $n$.

(2) $\widetilde{\perp}_{n} F^{h \Sigma_{n}}$ and $\widehat{\perp}_{n} F^{h \Sigma_{n}}$ are $(n-1)$-cohomogeneous degree $n$.

(3) $\operatorname{Tate}\left(\widetilde{\perp}_{n} F ; \Sigma_{n}\right)$ and Tate $\left(\widehat{\perp}_{n} F ; \Sigma_{n}\right)$ are degree $n-1$.

\section{MAIN RESULTS}

First note that, for a homotopy functor $F: S p e c \rightarrow S p e c$, the homotopy functor

$$
\widetilde{F}:=\operatorname{hofib}(F \rightarrow F(*))
$$

is clearly reduced (i.e. $\widetilde{F}(*) \simeq *$ ) and we also have

$$
F \simeq \widetilde{F} \vee F(*) \simeq \widetilde{F} \times F(*)
$$

Hence, without loss of generality, we will consider only reduced homotopy functors in this paper.

Let $F$ and $G$ be reduced homotopy functors of spectra. 
Definition 2.1. For $0 \leq r \leq n$, we define

$$
\begin{aligned}
& P_{n, r} F=\operatorname{hocofib}\left(p^{n-r} P_{n} F: P^{n-r} P_{n} F \rightarrow P_{n} F\right), \\
& Q_{n, r} F=\operatorname{hocofib}\left(q_{n} F \circ p^{n-r} P_{n} F: P^{n-r} P_{n} F \rightarrow P_{n-1} F\right), \\
& P^{n, r} F=\operatorname{hofib}\left(p_{n-r} P^{n} F: P^{n} F \rightarrow P_{n-r} P^{n} F\right), \\
& Q^{n, r} F=\operatorname{hofib}\left(p_{n-r} P^{n} F \circ q^{n} F: P^{n-1} F \rightarrow P_{n-r} P^{n} F\right) .
\end{aligned}
$$

Evidently, we obtain the following induced natural transformations:

$$
\begin{aligned}
& \text { - } s_{n, r} F: P_{n} F \rightarrow P_{n, r} F . \\
& \text { - } t_{n, r} F: P_{n-1} F \rightarrow Q_{n, r} F . \\
& \text { - } q_{n, r} F: P_{n, r} F \rightarrow Q_{n, r} F . \\
& \text { - } s^{n, r} F: P^{n, r} F \rightarrow P^{n} F . \\
& \text { - } t^{n, r} F: Q^{n, r} F \rightarrow P^{n-1} F . \\
& \text { - } q^{n, r} F: Q^{n, r} F \rightarrow P^{n, r} F .
\end{aligned}
$$

Also, we let

- $\epsilon_{n, r} F=s_{n, r} F \circ p_{n} F: F \rightarrow P_{n, r} F$.

- $\epsilon^{n, r} F=p^{n} F \circ s^{n, r} F: P^{n, r} F \rightarrow F$.

All definitions above can be summarized in the following commutative diagrams:

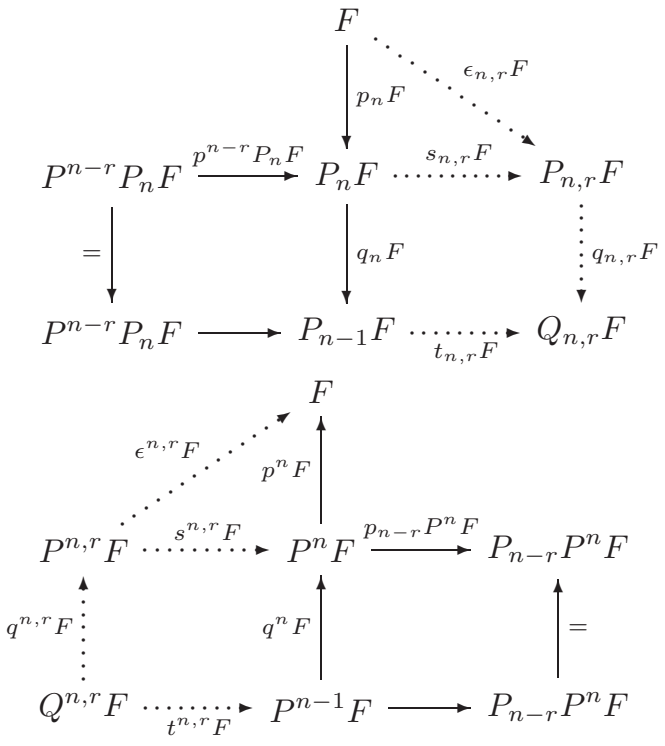

Remark 2.2. It is easy to verify that

- $P_{n, 0} F \simeq * \simeq P^{n, 0} F$.

- $Q_{n, 0} F \simeq \Sigma D_{n} F$.

- $Q^{n, 0} F \simeq \Sigma^{-1} D^{n} F$.

- $\operatorname{hofib}\left(q_{n, r} F\right) \simeq D_{n} F$.

- hocofib $\left(q^{n, r} F\right) \simeq D^{n} F$.

- $s_{n, n} F: P_{n} F \stackrel{\simeq}{\rightarrow} P_{n, n} F$.

- $t_{n, n} F: P_{n-1} F \stackrel{\simeq}{\rightrightarrows} Q_{n, n} F$.

- $s^{n, n} F: P^{n, n} F \stackrel{\simeq}{\leftrightarrows} P^{n} F$.

- $t^{n, n} F: Q^{n, n} F \stackrel{\cong}{\rightrightarrows} P^{n-1} F$. 
Lemma 2.3. For $0 \leq r \leq n$, we have

(1) $P_{n, r} F$ is $(n-r)$-cohomogeneous degree $n$.

(2) $P^{n, r} F$ is $(n-r)$-homogeneous degree $n$.

(3) If $r>0$, both $Q_{n, r} F$ and $Q^{n, r} F$ are degree $n-1$.

Proof. We will only prove (1) and (3), and leave (2) as an exercise.

By applying the $P^{n-r}$ to the cofibration sequence defining $P_{n, r} F$, i.e.,

$$
P^{n-r} P_{n} F \rightarrow P_{n} F \rightarrow P_{n, r} F
$$

we obtain the cofibration sequence

$$
P^{n-r} P^{n-r} P_{n} F \stackrel{\simeq}{\rightarrow} P^{n-r} P_{n} F \rightarrow P^{n-r} P_{n, r} F,
$$

where the left map becomes an equivalence.

Hence, $P^{n-r} P_{n, r} F \simeq *$; i.e., $P_{n, r} F$ is $(n-r)$-cohomogeneous.

Since a cross effect always commutes with a cofibration sequence, by applying $c r_{n+1}$ to the cofibration sequence defining $P_{n, r} F$, we obtain the cofibration sequence

$$
c r_{n+1} P^{n-r} P_{n} F \rightarrow c r_{n+1} P_{n} F \rightarrow c r_{n+1} P_{n, r} F .
$$

Since $P^{n-r} P_{n} F$ is degree $n-r$ and $P_{n} F$ is degree $n$, it follows that

$$
c r_{n+1} P^{n-r} P_{n} F \simeq * \simeq c r_{n+1} P_{n} F
$$

and hence $c r_{n+1} P_{n, r} F \simeq *$; i.e., $P_{n, r} F$ is degree $n$.

For (3), suppose $r>0$. By applying $c r_{n}$ to the cofibration sequence defining $Q_{n, r} F$, we obtain the cofibration sequence

$$
c r_{n} P^{n-r} P_{n} F \rightarrow c r_{n} P_{n-1} F \rightarrow c r_{n} Q_{n, r} F \text {. }
$$

Now, since $P^{n-r} P_{n} F$ is degree $n-r(\leq n-1)$ and $P_{n-1} F$ is degree $n-1$, it follows that

$$
c r_{n} P^{n-r} P_{n} F \simeq * \simeq c r_{n} P_{n-1} F
$$

and hence $c r_{n} Q_{n, r} F \simeq *$; i.e., $Q_{n, r} F$ is degree $n-1$.

Similarly, $Q^{n, r} F$ is also degree $n-1$.

Lemma 2.4. Suppose $F$ and $G$ are degree $n$, and $\eta: F \rightarrow G$ is a natural transformation.

(1) If $F$ is $(n-1)$-homogeneous and hocofib $(\eta)$ is degree $n-1$, then $F \simeq D_{n} G$ and $\operatorname{hocofib}(\eta) \simeq P_{n-1} G$.

(2) If $G$ is $(n-1)$-cohomogeneous and hofib $(\eta)$ is degree $n-1$, then $G \simeq D^{n} F$ and $\operatorname{hofib}(\eta) \simeq P^{n-1} F$. 
Proof. We will only prove (1), which is done by chasing the following commutative diagram where each row and column are fibration sequences:

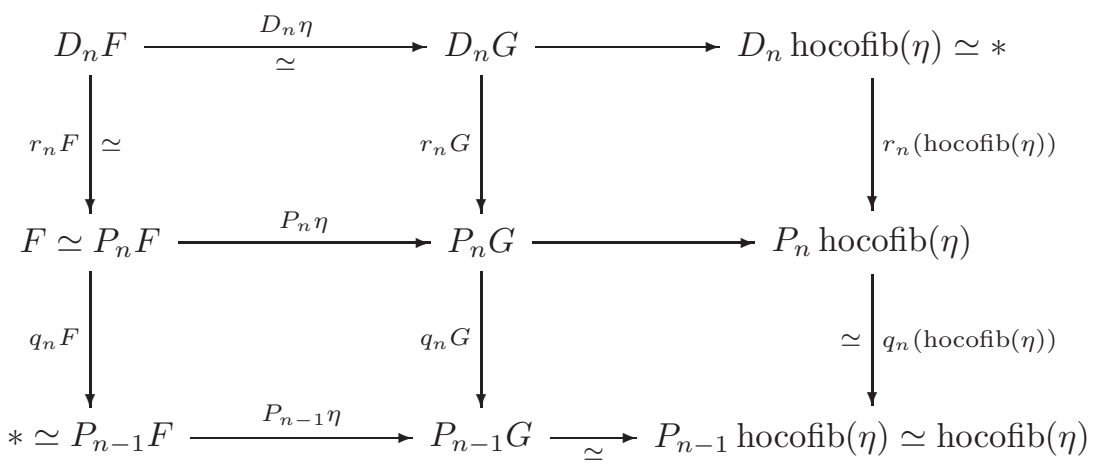

Theorem 2.5. For $0<r \leq n$, we have

(1) $Q_{n, r} F \simeq P_{n-1} P_{n, r} F$.

(2) $Q^{n, r} F \simeq P^{n-1} P^{n, r} F$.

Proof. By Remark 2.2 we have the cofibration sequence

$$
D_{n} F \simeq \operatorname{hofib}\left(q_{n, r} F\right) \rightarrow P_{n, r} F \stackrel{q_{n, r} F}{\longrightarrow} Q_{n, r} F .
$$

Note that $D_{n} F$ is generally known to be $(n-1)$-homogeneous degree $n$.

Since $P_{n, r} F$ is degree $n$ and $Q_{n, r} F$ is degree $n-1$ (by Lemma 2.3), it follows from the previous lemma that $Q_{n, r} F \simeq P_{n-1} P_{n, r} F$.

Similarly, it can be proved that $Q^{n, r} F \simeq P^{n-1} P^{n, r} F$.

Lemma 2.6. There is a commutative diagram

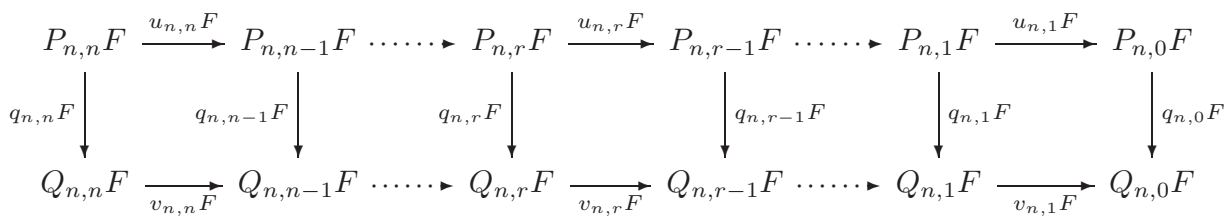

where each square is a homotopy pullback.

Proof. First note that each commutative square in the diagram above arises as the homotopy cofiber of the left cube in Figure 3, where $u_{n, r} F$ and $v_{n, r} F$ denote the induced maps.

Since the left cube is clearly co-cartesian, the rightmost square is then a homotopy pushout (which is equivalent to a homotopy pullback).

Lemma 2.7. There is a commutative diagram

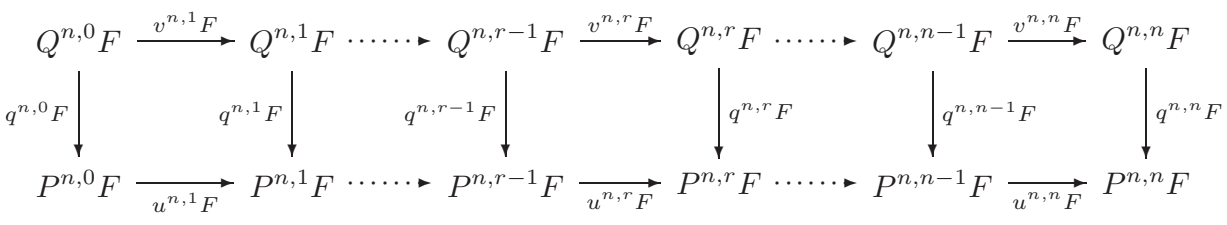

where each square is a homotopy pullback. 


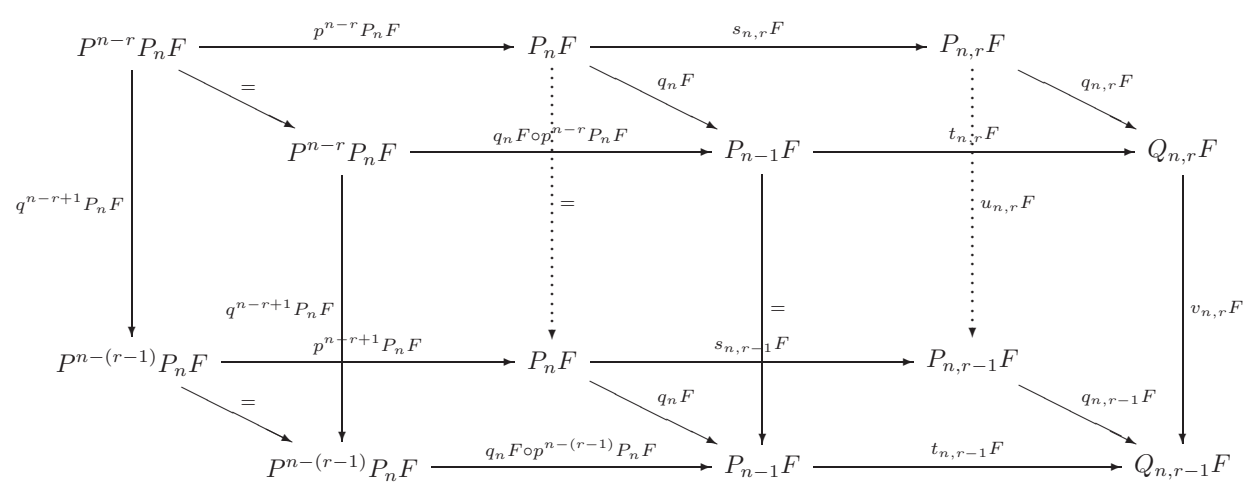

FiguRE 3.

Proof. Dualize the proof in Lemma 2.6 .

Definition 2.8. We say that a functor $F: S p e c \rightarrow$ Spec n-splits at $X \in \mathcal{C}$, where $n \geq 1$, if there exists a map $\alpha: P_{n} F(X) \rightarrow D_{n} F(X)$ such that the following square is a homotopy pullback:

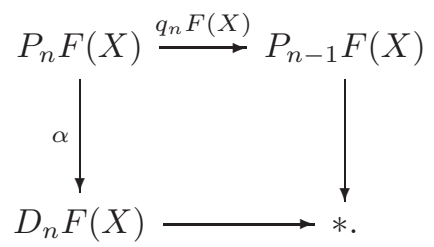

Dually, we say that $F n$-co-splits at $X \in$ Spec if there exists a map $\beta: D^{n} F(X) \rightarrow$ $P^{n} F(X)$ such that the following square is a homotopy pushout:

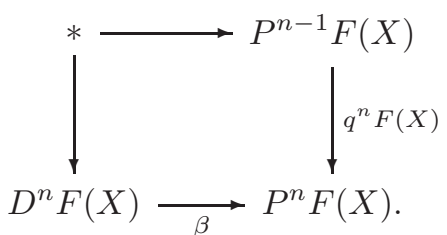

Remark 2.9. Every reduced homotopy functor of spectra automatically 1-splits and 1-co-splits.

Theorem 2.10. Let $X \in$ Spec.

(1) If $Q_{n, r} F(X) \simeq *$ for some $0 \leq r \leq n$, then $F$ n-splits at $X$.

(2) If $Q^{n, r} F(X) \simeq *$ for some $0 \leq r \leq n$, then $F$ n-co-splits at $X$.

Proof. We will only prove (1). 
If $n=r$, it is trivial. Otherwise, if $Q_{n, r} F(X) \simeq *$, we obtain the following homotopy pullback square from Lemma 2.6:

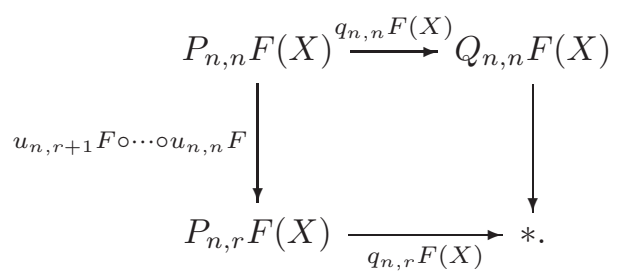

Since $P_{n, n} F(X) \simeq P_{n} F(X)$ and $Q_{n, n} F(X) \simeq P_{n-1} F(X)$, it follows that $P_{n, r} F(X)$ $\simeq D_{n} F(X)$, and hence, $F n$-splits at $X$.

Theorem 2.11. Let $X \in$ Spec.

(1) If $P_{n, r} F n$-splits at $X$ for some $0 \leq r \leq n$, then so does $F$.

(2) If $P^{n, r} F n$-co-splits at $X$ for some $0 \leq r \leq n$, then so does $F$.

Proof. We will only prove (1). If $n=r$, it is trivial.

Otherwise, if $P_{n, r} F n$-splits at $X$, then, together with Lemma 2.6, we have the following commutative diagram, for some $\alpha: P_{n, r} F(X) \rightarrow D_{n} P_{n, r} F(X)$ :

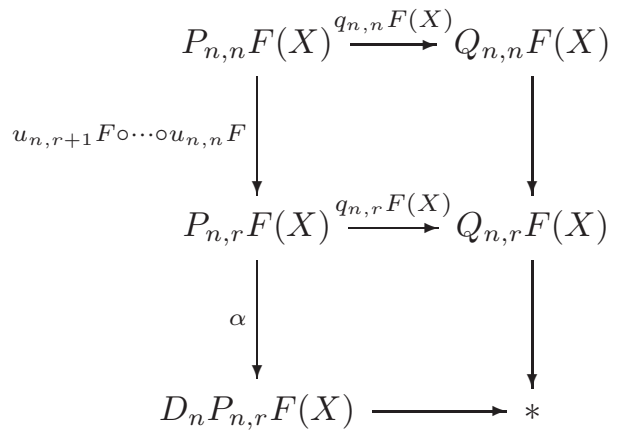

where each square is a homotopy pullback. Also, we have $P_{n, n} F(X) \simeq P_{n} F(X)$ and $Q_{n, n} F(X) \simeq P_{n-1} F(X)$. From Definition 2.1 it is easy to see that $D_{n} F \simeq$ $D_{n} P_{n, r} F$, and hence, the outer square implies that $F n$-splits.

\section{Applichtions}

In this section, we will show that the splitting criteria in [6] are special cases of Theorem 2.10 above. As before, we will let $F:$ Spec $\rightarrow$ Spec be a reduced homotopy functor.

Theorem 3.1. We have the following equivalences:

(1) $P_{n, 1} F \simeq D^{n} P_{n} F$.

(2) $D^{n} P_{n} F \simeq \tilde{\perp}_{n} F^{h \Sigma_{n}}$.

(3) $Q_{n, 1} F \simeq \operatorname{Tate}\left(\widetilde{\perp}_{n} F ; \Sigma_{n}\right)$. 
In particular, if $C_{n}$ is the $n$-th derivative of the functor $F$, we have a homotopy pullback square

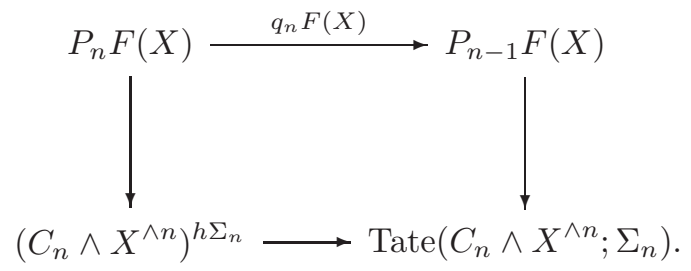

Proof. For (1), consider the following commutative diagram where each row is a cofibration sequence:

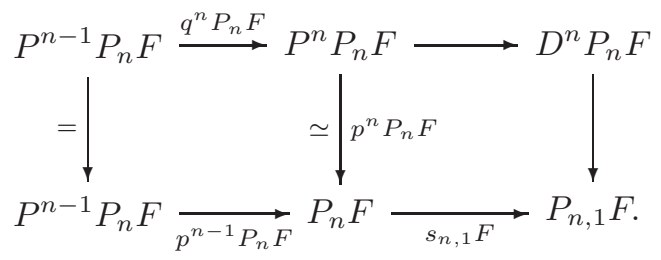

Since the left and the middle vertical maps are equivalences (and our target category is $S p e c$ ), so is the right one.

For (2), first note that we have $D^{n} P_{n} F \simeq D^{n} D_{n} F$ from the following cofibration sequence:

$$
D^{n} D_{n} F \stackrel{\simeq}{\rightarrow} D^{n} P_{n} F \rightarrow D^{n} P_{n-1} F \simeq * .
$$

Now, consider the cofibration sequence (induced by the Tate sequence)

$$
\Sigma^{-1} \operatorname{Tate}\left(\widetilde{\perp}_{n} F ; \Sigma_{n}\right) \rightarrow \widetilde{\perp}_{n} F_{h \Sigma_{n}} \stackrel{t}{\rightarrow} \widetilde{\perp}_{n} F^{h \Sigma_{n}} .
$$

Since $\widetilde{\perp}_{n} F_{h \Sigma_{n}} \simeq D_{n} F$ is degree $n$ and $\widetilde{\perp}_{n} F^{h \Sigma_{n}}$ is $(n-1)$-cohomogeneous degree $n$ and $\Sigma^{-1}$ Tate $\left(\widetilde{\perp}_{n} F ; \Sigma_{n}\right)$ is degree $n-1$, we have $D^{n} D_{n} F \simeq \widetilde{\perp}_{n} F^{h \Sigma_{n}}$ by Lemma 2.4 (2).

For (3), it follows from Theorem 2.5 and (1) and (2) above that

$$
Q_{n, 1} F \simeq P_{n-1} P_{n, 1} F \simeq P_{n-1}\left(\widetilde{\perp}_{n} F^{h \Sigma_{n}}\right) .
$$

Now, consider the Tate sequence

$$
D_{n} F \simeq \widetilde{\perp}_{n} F_{h \Sigma_{n}} \stackrel{t}{\rightarrow} \widetilde{\perp}_{n} F^{h \Sigma_{n}} \rightarrow \operatorname{Tate}\left(\widetilde{\perp}_{n} F ; \Sigma_{n}\right)
$$

Since $\widetilde{\perp}_{n} F_{h \Sigma_{n}}$ is $(n-1)$-homogeneous degree $n$ and $\widetilde{\perp}_{n} F^{h \Sigma_{n}}$ is degree $n$ and $\operatorname{Tate}\left(\widetilde{\perp}_{n} F ; \Sigma_{n}\right)$ is degree $n-1$, we have $P_{n-1}\left(\widetilde{\perp}_{n} F^{h \Sigma_{n}}\right) \simeq \operatorname{Tate}\left(\widetilde{\perp}_{n} F ; \Sigma_{n}\right)$ by Lemma 2.4 (1).

Corollary 3.2. Let $X \in$ Spec.

(1) If Tate $\left(\widetilde{\perp}_{n} F(X) ; \Sigma_{n}\right) \simeq *$, then $F$ n-splits at $X$; i.e.,

$$
P_{n} F(X) \simeq P_{n-1} F(X) \times D_{n} F(X) .
$$

(2) If Tate $\left(\widetilde{\perp}_{i} F(X) ; \Sigma_{i}\right) \simeq *$ for $i>n$ and the tower of $F$ converges at $X$, then

$$
F(X) \simeq P_{n} F(X) \times \prod_{i=n+1}^{\infty} D_{i} F(X) .
$$


The theorem and corollary above can be dualized to get the similar results for cotowers.

Theorem 3.3. We have the following equivalences:

(1) $P^{n, 1} F \simeq D_{n} P^{n} F$.

(2) $D_{n} P^{n} F \simeq \widehat{\perp}_{n} F_{h \Sigma_{n}}$.

(3) $Q^{n, 1} F \simeq \Sigma^{-1} \operatorname{Tate}\left(\widehat{\perp}_{n} F ; \Sigma_{n}\right)$.

Corollary 3.4. Let $X \in$ Spec.

(1) If Tate $\left(\widehat{\perp}_{n} F(X) ; \Sigma_{n}\right) \simeq *$, then $F$-co-splits at $X$; i.e.,

$$
P^{n} F(X) \simeq P^{n-1} F(X) \times D^{n} F(X) .
$$

(2) If Tate $\left(\widehat{\perp}_{i} F(X) ; \Sigma_{i}\right) \simeq *$ for $i>n$ and the cotower of $F$ converges at $X$, then

$$
F(X) \simeq P^{n} F(X) \times \prod_{i=n+1}^{\infty} D^{i} F(X) .
$$

\section{ACKNOWLEDGEMENT}

The author wishes to thank Randy McCarthy for reading and providing many helpful comments on the very first version of this paper.

\section{REFERENCES}

1. P. Chaoha, Obstructions to constructing the Taylor tower of finite degree functors of spectra, Ph.D. thesis, UIUC, May 2001.

2. T. Goodwillie, Calculus I: The first derivative of pseudoisotopy theory, K-Theory 4 (1990), 1-27. MR 92m:57027

3. T. Goodwillie, Calculus II: Analytic functors, K-Theory 5 (1992), 295-332. MR 93i:55015

4. T. Goodwillie, Calculus III: Taylor series of a homotopy functor, in preparation.

5. B. Johnson and R. McCarthy, Deriving calculus with cotriples, Trans. Amer. Math. Soc. 356 (2004), 757-803.

6. R. McCarthy, Dual calculus for functors to spectra, Homotopy methods in algebraic topology (Boulder, CO, 1999), Contemp. Math., 271, Amer. Math. Soc., Providence, RI, 2001, pp. 183-215. MR 2002c:18009

Department of Mathematics, Faculty of Science, Chulalongkorn University, BANGKOK 10330, THAILAND

E-mail address: phichet.c@chula.ac.th 